DESY $10-114$

\title{
Chameleon Induced Atomic Afterglow
}

\author{
Philippe Brax ${ }^{1}$ and Clare Burrage ${ }^{2}$ \\ ${ }^{1}$ Institut de Physique Théorique, CEA, IPhT, CNRS, URA2306, F-91191 \\ Gif-sur-Yvette cédex, France \\ 2 Theory Group, Deutsches Elektronen-Synchrotron DESY, D-22603, Hamburg, \\ Germany \\ E-mail: philippe.brax@cea.fr, clare.burrage@desy.de
}

\begin{abstract}
The chameleon is a scalar field whose mass depends on the density of its environment. Chameleons are necessarily coupled to matter particles and will excite transitions between atomic energy levels in an analogous manner to photons. When created inside an optical cavity by passing a laser beam through a constant magnetic field, chameleons are trapped between the cavity walls and form a standing wave. This effect will lead to an afterglow phenomenon even when the laser beam and the magnetic field have been turned off, and could be used to probe the interactions of the chameleon field with matter.
\end{abstract}




\section{Introduction}

Light scalar fields are commonly invoked as the solution to various cosmological problems, including the origins of dark energy and inflation. They are also generic in theories of beyond the standard model particle physics. Problematically, these light scalar fields will also mediate new fifth forces which have not yet been seen and are very tightly constrained by experiments [1].

In [2, 3] Khoury and Weltman proposed a mechanism by which light scalar fields could evade gravitational tests of fifth forces. If the scalar field couples to matter in a non-minimal way then non-linearities in the theory mean that the mass of the scalar field becomes dependent on its environment. By becoming heavy in dense environments and light in diffuse ones the scalar field avoids experimental probes of gravity thanks to what is known as the thin shell mechanism. We shall call scalar fields which behave in this way chameleon $\ddagger$.

The dynamical way in which chameleonic scalar fields avoid detection in experiments means that their coupling to matter fields can be as strong as, or stronger than, the gravitational coupling [7]. This invites us to consider ways in which the chameleon could be detected, in particular those experiments conducted in near vacuum where the chameleon cannot hide by adjusting its mass. Many constraints on the properties of the chameleon have been obtained in this way by proposing an additional coupling of the scalar field to photons [8], and such a coupling was shown to be generic in extensions of the standard model [9]. A coupling between photons and chameleons allows for many new searches for the chameleon both in the laboratory [8, 10, 11, 12, 13, 14, 15, 9, 16] and with astrophysics [17, 18, 19, 20, 21, 22, 23].

Of particular interest for this work are the laboratory searches known as afterglow experiments [11, 12, 13. In such experiments a vacuum tube is placed into a magnetic field, and a laser beam is shone through the tube. In the presence of the magnetic field there is a probability $P_{\gamma \leftrightarrow \phi}$ that a photon in the laser light may oscillate into a chameleon particle [24]. The chameleons produced in this way cannot exit from the vacuum tube, as they do not have enough energy to adjust their mass sufficiently to pass through the wall. Once the laser source has been turned off we are left with a vacuum tube full of chameleons. However if the magnetic field remains, there is still a probability $P_{\gamma \leftrightarrow \phi}$ that the chameleon will convert back into a photon. These reconverted photons are known as the afterglow and can be detected. Afterglow searches for chameleonic fields have been performed with optical photons by GammeV [13, 14] and with microwave photons by ADMX [16].

If chameleons are produced and their effects detected in afterglow experiments we will have a good probe of their mass and the strength of their coupling to photons. A complete theory of the chameleon also requires knowledge of their coupling to matter

¥ There exist related models of scalar fields with environment dependent properties [4, 5] that avoid the constraints of fifth force experiments [6] but do not exhibit the afterglow phenomenon discussed in this article. 
fields. This coupling is currently only weakly constrained by gravitational experiments [7] and probes of the Casimir force [25]. In this article we describe a new experiment to probe the coupling of the chameleon to matter.

We will show that the chameleon can excite transitions between atomic energy levels, in a similar manner to the usual photon induced transitions. However as the chameleon is a scalar field, whilst the photon is a vector field, different transitions will be excited by the two fields. In this article we focus on the scenario in which an electron is excited from a lower to a higher energy level by a chameleon field, and then decays to a lower energy level by emission of a photon. The detection of these photons would allow the coupling of the chameleon to photons to be probed directly.

The paper is organized as follows. In a first section, we describe the interaction of the chameleons with bound electrons in atoms. We then solve the two level system when bound electrons hop between two atomic levels due to the chameleon interaction. We show that for most experimental situations, the chameleon-matter interaction is weak and that the end result of the chameleon interaction with bound electrons is the creation of a long lived photonic afterglow.

\section{The Chameleon and its Interaction with Matter}

\subsection{Chameleon Models}

The simplest action for a chameleon field, $\phi$, is [2, 3]

$$
S=\int d^{4} x \sqrt{-g}\left\{\frac{M_{P}^{2}}{2} R-\frac{1}{2}(\partial \phi)^{2}-V(\phi)\right\}-\int d^{4} x \mathcal{L}_{m}\left(\psi^{(i)}, \tilde{g}_{\mu \nu}\right),
$$

where the matter fields $\psi^{(i)}$ feel the Jordan frame metric $\tilde{g}$ which is related to the Einstein frame metric $g$ by

$$
\tilde{g}_{\mu \nu}=e^{2 \beta \phi} g_{\mu \nu} .
$$

$V(\phi)$ is an arbitrary scalar potential. The Einstein and Jordan frame descriptions are completely equivalent classically. In the Einstein picture the masses and couplings of elementary particles become dependent on the chameleon field. With such a conformal coupling (2) there is no tree level interaction between the scalar field and photons, however we are allowed to add such a term to the action

$$
S_{\gamma}=\int d^{4} x \sqrt{-g} \phi \beta_{\gamma} F_{\mu \nu} F^{\mu \nu}
$$

the existence of which follows from quantum effects [9].

When the equations of motion for the fields are computed from the action (1), it becomes apparent that the dynamics of the chameleon are governed by an effective potential

$$
V_{e f f}(\phi)=V(\phi)-e^{4 \beta \phi} \tilde{T}_{\mu}^{\mu}
$$


where $\tilde{T}_{\nu}^{\mu}$ is the usual Jordan frame energy momentum tensor for matter fields. We restrict our attention to the behaviour of the chameleon in the presence of non-relativistic matter for which $\tilde{T}_{\mu}^{\mu}=-\rho$, and $\rho$ is the energy density.

For the chameleon mechanism to function the bare potential, $V(\phi)$, must be chosen so that the effective potential has a minimum, $V_{e f f}^{\prime}\left(\phi_{m}\right)=0$, and the position of this minimum is a function of the local energy density. The chameleon mechanism also requires that the mass of small fluctuations about this minimum

$$
m^{2}=V^{\prime \prime}\left(\phi_{m}\right)+\beta^{2} \rho e^{4 \beta \phi},
$$

is a monotonic function of the local energy density, and that the mass of the chameleon field increases with increasing density.

The most stringent constraints on $\beta$ come from measurements of the Casimir force [25]. For potentials of the form $V=\Lambda_{0}^{4}\left[1+(\Lambda / \phi)^{n}\right]$ where $|n| \sim \mathcal{O}(1)$ and $\Lambda_{0}=\Lambda=2.4 \times 10^{3} \mathrm{eV}$ the experiments constrain $\beta \mathrm{GeV} \gtrsim 10^{-16}$. For other forms of the potential the constraints on $\beta$ are less stringent. Constraints from particle physics experiments, [15], give $\beta \mathrm{GeV} \lesssim 10^{-4}$.

\subsection{Chameleon-Atom Interactions}

In this article we describe a new technique to probe the coupling of the chameleon to matter fields if they are produced in afterglow experiments. The coupling of the chameleon to the matter fields in the action (11), implies an interaction between a background chameleon field and electrons residing in atoms. The existence of such a coupling means that a chameleon field can excite atomic energy level transitions, in the same way that a background photon field can give rise to atomic absorption and emission. To study how the chameleon field can excite electrons and induce transitions between energy levels we begin by deriving the perturbed Hamiltonian governing the behaviour of the electrons in the presence of a chameleon field.

The Dirac equation for a two component spinor is

$$
i \frac{\partial}{\partial t}\left(\begin{array}{l}
\chi_{+} \\
\chi_{-}
\end{array}\right)=\left(\begin{array}{cc}
m(\phi) & \sigma \cdot p \\
\sigma \cdot p & -m(\phi)
\end{array}\right)\left(\begin{array}{l}
\chi_{+} \\
\chi_{-}
\end{array}\right)
$$

where the mass of the spinor is dependent on the value of the chameleon field. Under the transformation

$$
\left(\begin{array}{c}
\chi_{+} \\
\chi_{-}
\end{array}\right)=\exp \left(-i \int m d t\right)\left(\begin{array}{c}
\Phi \\
X
\end{array}\right)
$$

the equations of motion become

$$
\begin{aligned}
& i \dot{\Phi}=e^{i \int m d t} \sigma \cdot p\left(e^{-i \int m d t} X\right), \\
& i \dot{X}=e^{i \int m d t} \sigma \cdot p\left(e^{-i \int m d t} \Phi\right)-2 m X,
\end{aligned}
$$

and we impose that $X$ does not vary significantly with time:

$$
X=\frac{1}{2 m} e^{i \int m d t} \sigma \cdot p\left(e^{-i \int m d t} \Phi\right) .
$$


A final transformation $\psi=e^{-i \int m d t} \Phi$ gives the Schrodinger-type equation

$$
i \dot{\psi}=\left[\frac{p^{2}}{2 m}+m(\phi)+\frac{1}{2}(\sigma \cdot p)\left(\frac{1}{m(\phi)}\right)(\sigma \cdot p)\right] \psi .
$$

Assuming that the variation of $\phi$ about its background value $\phi_{0}$ is small we can approximate the mass of the electron $m(\phi)=m_{e}(1+\beta \delta \phi)$. Then to first order in the chameleon fluctuation the Hamiltonian becomes

$$
H=\frac{p^{2}}{2 m_{e}}+m_{e}-\frac{\beta}{2 m_{e}}\left[\delta \phi p^{2}+(\sigma \cdot p) \delta \phi(\sigma \cdot p)\right]+m_{e} \beta \delta \phi .
$$

\subsection{Transitions Between Energy Levels}

The chameleon perturbation of the electron Hamiltonian, derived in the previous section, allows electrons to have transitions from one energy level of an atom to another. If $|i\rangle$ is the i-th excited state of an atom the transition rate between one energy level and another due to the chameleon is $\langle i|\delta H(\phi)| j\rangle$, where

$$
\delta H=-\frac{\beta}{2 m_{e}}\left[\phi p^{2}+(\sigma \cdot p) \phi(\sigma \cdot p)\right]+m_{e} \beta \phi,
$$

and we now denote the chameleon fluctuations by $\phi$.

In a vacuum tube, aligned along the $z$ axis, that has been filled with chameleons by conversion from photons with frequency $\bar{\omega}$, the chameleon field can be written as

$$
\phi(t, z)=-a \cos \bar{\omega} t \cos \bar{\omega} z,
$$

where $a$ is a constant that will be determined in Section 2.4.

The wave-functions describing the energy levels of an hydrogenic atom are exponentially suppressed outside the Bohr radius of the atom, $a_{0}$. Therefore it is sufficient to expand the chameleon wave function about the position of an atom, $z_{0}$, on the $z$-axis

$$
\phi\left(t, z_{0}+r \cos \theta\right)=-a \cos \bar{\omega} t \times\left(\cos \bar{\omega} z_{0}-\bar{\omega} r \cos \theta \sin \bar{\omega} z_{0}\right) .
$$

Where $r, \theta$ and $\phi$ are spherical polar coordinates centered at the nucleus of the atom. We have assumed $r$ is small compared to the scale of variation in the chameleon wavefunction.

We consider transitions between the 1 s and 2 p energy levels of a hydrogenic atom, with nuclear charge $Z e$ \&. The wave functions for these states are (in spherical polar coordinates)

$$
\begin{aligned}
& \psi_{1 s}=\frac{1}{\sqrt{\pi}}\left(\frac{Z}{a_{0}}\right)^{3 / 2} e^{-Z r / a_{0}} \\
& \psi_{2 p}=\frac{1}{\sqrt{\pi}}\left(\frac{Z}{2 a_{0}}\right)^{5 / 2} e^{-Z r / 2 a_{0}} r \cos \theta
\end{aligned}
$$

$\S$ The chameleon can also induce transitions from the $1 \mathrm{~s}$ to the $2 \mathrm{~s}$ level. This transition is optically forbidden as optical transitions are mostly due to a dipole interaction which changes the parity of the wave functions. 
An order of magnitude estimate for the size of $\langle i|\delta H(\phi)| j\rangle$, with $H(\phi)$ given in (12), shows that the term due to $m_{e} \beta \phi$ will always dominate over those that are a function of the momenta. The transition probabilities due to this term in the Hamiltonian are

$$
\begin{aligned}
\left\langle 1,0\left|m_{e} \beta \phi\right| 2,1\right\rangle & =\frac{2^{8} \pi}{\sqrt{2} 3^{5} Z} m_{e} \beta a_{0} \bar{\omega} a \sin \bar{\omega} z_{0} \cos \bar{\omega} t, \\
\left\langle 1,0\left|m_{e} \beta \phi\right| 1,0\right\rangle & =\left\langle 2,1\left|m_{e} \beta \phi\right| 2,1\right\rangle=-m_{e} \beta a \cos \bar{\omega} z_{0} \cos \bar{\omega} t .
\end{aligned}
$$

\subsection{Chameleons in a Cavity}

To fully calculate the transition probabilities induced by the chameleon we need to know the wavefunction describing the behaviour of chameleons in a cavity. We envisage a scenario where the cavity has been filled with a bath of chameleons by an afterglowlike experiment. A simplified form of the set up has the photons entering the tube at $z=0$ and passing straight through to exit at $z=L$. The chameleons are reflected at $z=0, L$.

Following the derivation of [1] and assuming that, as we are dealing with laser sources, the incoming photon beam is very sharply peaked about the frequency $\omega=\bar{\omega}$, we find the following expression for the chameleon field inside the cavity

$$
\begin{aligned}
\phi(t, z)= & -i \vartheta e^{-i \bar{\omega}(t-z)} \alpha(\bar{\omega}) \\
& \times\left(1+\frac{\bar{\omega} e^{-i \bar{\omega} z}}{k_{+} \sin k_{+} L}\left(\sin k_{+}(z-L)+e^{i \bar{\omega} L} \sin k_{+} z\right)\right)+\mathcal{O}\left(\vartheta^{2}\right),
\end{aligned}
$$

where $\vartheta$ is the angle describing the mixing between chameleons and photons, which is assumed to be small; $\vartheta \approx \bar{\omega} B \beta_{\gamma} / m^{2} M_{\mathrm{P}}$ [24]. Here $m$ is the mass of the chameleon inside the cavity and $B$ the strength of the magnetic field. $\alpha(\omega)$ is the amplitude of oscillations of the incoming photons, and $k_{+}=\bar{\omega}-m^{2} / 2 \bar{\omega}$.

Once a chameleon field is present in the cavity the magnetic field is turned off at time $t=0$. The chameleons now propagate freely inside the cavity. The solution for the chameleon wavefunction at time $t>0$ can be found by Fourier expanding the wavefunction and matching with (20) at time $t=0$. The standing wave solution is

$$
\phi=-2 \vartheta \bar{\omega} L \alpha(\bar{\omega}) \sin \bar{\omega} L \sum_{n \geq 0} \frac{\cos \omega_{n} t \cos k_{n} z}{\pi^{2} n^{2}-k_{+}^{2} L^{2}}\left(1+(-1)^{n} \cos k_{+} L\right),
$$

where $k_{n} L=n \pi$ and $k_{n}^{2}=\omega_{n}^{2}-m^{2}$. As $k_{+}=\bar{\omega}\left(1+\mathcal{O}\left(m^{2} / \bar{\omega}^{2}\right)\right)$ and we assume that $m \ll \bar{\omega}$, as is the case for the GammeV experiment. The dominant contributions are the terms with $|n| \sim \bar{\omega} L$. Assuming that the length of the cavity is not tuned to be resonant with the optical frequency $\bar{\omega}^{2} L^{2}-n^{2} \pi^{2}$ never vanishes, and the leading order behaviour of the chameleon wavefunction is

$$
\phi(t, z)=-a \cos \bar{\omega} t \cos \bar{\omega} z,
$$

where $a=a_{-}+a_{+}$together with

$$
a_{ \pm}=\frac{4 \vartheta \bar{\omega} L \alpha(\bar{\omega})}{\left(\bar{\omega}^{2} L^{2}-N^{2} \pi^{2}\right)} \sin \bar{\omega} L(1 \mp \cos \bar{\omega} L),
$$

and $N$ is the closest integer to $\bar{\omega} L / \pi$. 


\section{The Two State System}

\subsection{Evolution of the Electron Bound State}

We study a simplified system in which an atom has only two energy levels. This is a good approximation to the excitation of the ground state of an atom when there is a unique gap between levels that is close to the energy of the chameleon field, however we do not require an exact resonance. The approximation of a two state system breaks down if the number of excited states with an energy gap close to the chameleon energy is greater than one. We label the lower and upper levels 1 and 2 respectively. The electron wavefunction is described by $|\psi\rangle=c_{1}|1\rangle+c_{2}|2\rangle$, where $c_{1}$ and $c_{2}$ are the probability amplitudes that the electron will be found in the first and second energy level respectively. For full discussion of photon induced transitions in a two state system see, for example, 26].

The coefficients $c_{i}$ evolve with time according to

$$
\frac{\partial}{\partial t}\left(\begin{array}{l}
c_{1} \\
c_{2}
\end{array}\right)=i a \cos \bar{\omega} t\left(\begin{array}{cc}
A & -B e^{-i \omega_{0} t} \\
-B e^{i \omega_{0} t} & A
\end{array}\right)\left(\begin{array}{l}
c_{1} \\
c_{2}
\end{array}\right),
$$

where $\omega_{0}$ is the energy difference between the two energy levels under consideration, for two energy levels of a hydrogenic atom $\omega_{0} \sim \mathcal{O}(\mathrm{eV})$. We have also defined

$$
\begin{aligned}
A & =m_{e} \beta \cos \bar{\omega} z_{0}, \\
B & =\frac{2^{8} m_{e} \beta a_{0} \bar{\omega} \pi}{\sqrt{2} 3^{5} Z} \sin \bar{\omega} z_{0} .
\end{aligned}
$$

Writing

$$
\begin{aligned}
& c_{1}=\tilde{c}_{1} \exp \left(-\frac{i A a}{\bar{\omega}} \sin \bar{\omega} t\right), \\
& c_{2}=\tilde{c}_{2} \exp \left(-\frac{i A a}{\bar{\omega}} \sin \bar{\omega} t\right),
\end{aligned}
$$

the equations can be diagonalised

$$
\frac{\partial}{\partial t}\left(\begin{array}{c}
\tilde{c}_{1} \\
\tilde{c}_{2}
\end{array}\right)=-i a B \cos \bar{\omega} t\left(\begin{array}{cc}
0 & e^{-i \omega_{0} t} \\
e^{i \omega_{0} t} & 0
\end{array}\right)\left(\begin{array}{c}
\tilde{c}_{1} \\
\tilde{c}_{2}
\end{array}\right) .
$$

As is the case for photon driven excitations, there are two limits in which these equations can be solved analytically, called the weak and strong field limits. We discuss these in detail in the following sections.

\subsection{The Weak Field Limit}

If the electron-chameleon coupling is weak then the number of atoms in the lowest energy level is always much greater than the number in the excited energy level. The approximations $\tilde{c}_{2} \ll \tilde{c}_{1} \approx 1$, valid when $|A a| \ll \bar{\omega}$, reduce the system of equations to

$$
\frac{\partial \tilde{c}_{2}}{\partial t}=i a B e^{i \omega_{0} t} \cos \bar{\omega} t
$$


To integrate this equation we must recall that both the spectral line and the laser beam have a finite spectral width; in the evaluation of $a$ there is an integral over frequency which we have assumed to be infinitely sharply peaked at $\omega=\bar{\omega}$. Replacing $a^{2}=\int u(\omega) d \omega$ allows us to integrate over the spectrum of the laser beam, where we choose the laser profile to be approximated with a Lorentzian distribution of width $\Delta \omega$,

$$
u(\omega)=\frac{a^{2} \Delta \omega}{\pi} \frac{1}{(\omega-\bar{\omega})^{2}+\Delta \omega^{2}} .
$$

The spectral line also has a Lorentzian distribution centered on the frequency $\omega_{0}$ with width $\Delta \omega_{0}$. We deduce that:

$$
\left|\tilde{c}_{2}\right|^{2}=B^{2} \int_{\omega_{0}-\Delta \omega_{0} / 2}^{\omega_{0}+\Delta \omega_{0} / 2} u(\omega)\left(\frac{\sin \left(\omega-\omega_{0}\right) t / 2}{\omega-\omega_{0}}\right)^{2} d \omega .
$$

We assume that $u(\omega)$ does not vary over the width of the spectral line, which is valid either when the laser is tuned to the resonance $\omega_{0}=\bar{\omega}$ and $\Delta \omega>\Delta \omega_{0}$, or when the spectral line occurs in the tail of the laser spectrum $\left|\bar{\omega}-\omega_{0}\right| \gg \Delta \omega$. Then we find

$$
\left|c_{2}(t)\right|^{2}=\frac{B^{2} \pi}{2} u\left(\omega_{0}\right) t
$$

after imposing the initial condition $c_{2}(0)=0$. The chameleon Einstein coefficient is

$$
B_{\text {chameleon }}=u\left(\omega_{0}\right) \frac{\pi\left\langle B^{2}\right\rangle}{2},
$$

where we have averaged over the position of the atom in the cavity

$$
\sqrt{\left\langle B^{2}\right\rangle}=\frac{2^{7} m_{e} \beta a_{0} \bar{\omega} \pi}{3^{5}}
$$

The excited level can be populated thanks to the chameleons and depleted due to photons. Therefore

$$
\frac{d N_{2}}{d t}=B_{\text {chameleon }} N_{1}-B_{\gamma} N_{2}
$$

due to the emission of photons, where $B_{\gamma}=\left(\pi / 3 \epsilon_{0} \hbar^{2}\right) \mu_{12}^{2}$ is the photonic Einstein coefficient and $\mu_{12}$ is the dipole matrix element. The photons leave the cavity very rapidly so we neglect photon stimulated emission. Similarly, the number of electrons in the ground state evolves according to

$$
\frac{d N_{1}}{d t}=-B_{\text {chameleon }} N_{1}
$$

as chameleons are the only particles permanently present in the cavity. Finally the number of emitted photons is

$$
\frac{d N_{\gamma}}{d t}=B_{\gamma} N_{2}
$$

We find that

$$
N_{1}=N_{1}(0) e^{-B_{\text {chameleon }} t} .
$$

We reach a steady state as long as $t \ll 1 / B_{\text {chameleon }}$ in which

$$
N_{2}=\frac{B_{\text {chameleon }}}{B_{\gamma}} N_{1}
$$


and the number of created photons per unit time is constant

$$
\frac{d N_{\gamma}}{d t}=B_{\text {chameleon }} N_{1} .
$$

Hence $B_{\text {chameleon }}$ gives the number of photons per unit time emitted by a single atom due to the interaction with chameleons. It depends on $u\left(\omega_{0}\right)$ crucially implying that if $\omega$ and $\omega_{0}$ are not close, the density $u\left(\omega_{0}\right)$ is going to be tiny. On the other hand if there is a quasi-resonance then we may create many photons.

\subsection{The Strong Field Limit}

In the limit of an exact resonance we can solve the equations of motion without restricting our attention to the situation where the perturbations due to the chameleon field are small. We again make a rotating wave approximation so that the two state system is described by

$$
\frac{\partial}{\partial t}\left(\begin{array}{c}
\tilde{c}_{1} \\
\tilde{c}_{2}
\end{array}\right)=-\frac{i a B}{2}\left(\begin{array}{cc}
0 & 1 \\
1 & 0
\end{array}\right)\left(\begin{array}{c}
\tilde{c}_{1} \\
\tilde{c}_{2}
\end{array}\right),
$$

with solution

$$
\begin{aligned}
& \tilde{c}_{1}(t)=\cos \left(\Omega_{R} t / 2\right), \\
& \tilde{c}_{2}(t)=i \sin \left(\Omega_{R} t / 2\right) .
\end{aligned}
$$

Where

$$
\begin{aligned}
\Omega_{R} & =|-a B| \\
& =\frac{2^{10} m_{e} \beta a_{0} \bar{\omega}^{2} L \alpha(\bar{\omega}) \vartheta}{\sqrt{2} 3^{5} Z\left(\bar{\omega}^{2} L^{2}-N^{2}\right)} \sin \bar{\omega} L \sin \bar{\omega} z_{0}(1 \mp \cos \bar{\omega} \pi L),
\end{aligned}
$$

is known as the Rabi frequency. The probability for finding the electron in the upper, or lower, level oscillates, a behaviour known as Rabi oscillations.

\subsection{Predictions for a GammeV-like Afterglow Experiment}

To see if either of the effects described above are detectable with current experimental set ups we specialize to the specifications of a simplified form of the Fermilab GammeV experiment. The GammeV experiment has a cavity of length $L=6 \mathrm{~m}$, and a laser beam of power of $160 \mathrm{~mJ}$ which operates at a frequency of $2.33 \mathrm{eV}$. The laser beam emits $5 \mathrm{~ns}$ wide pulses, and we assume a beam diameter of $5 \mathrm{~mm}$. This implies $\alpha_{0}=1.2 \times 10^{-8} \mathrm{GeV}$. We assume that the the atoms under consideration inside the cavity have $Z \sim 1$ and transition frequency $\omega_{0} \sim 1 \mathrm{eV}$. The Bohr radius of such hydrogenic atoms is $a_{0}=5.3 \times 10^{-11} \mathrm{~m}$. We assume that the cavity is not resonant with the optical frequency so that $\bar{\omega}^{2} L^{2}-N^{2} \pi^{2} \sim \mathcal{O}\left(10^{-1}\right)$.

We focus first on the possibility that observable Rabi oscillations occur. The Rabi frequency depends on the strength of the chameleon to matter coupling $\beta$ and the photon-chameleon oscillation angle $\vartheta$

$$
\Omega_{R} \sim 10^{19} \vartheta(\beta \mathrm{GeV}) \mathrm{s}^{-1},
$$


assuming the trigonometric terms in $a$ and $B$ are of order one. The GammeV experiment is sensitive to $\vartheta \gtrsim 10^{-9}$ so in the strong field limit of a GammeV-like experiment the chameleon induced Rabi frequency satisfies $\Omega_{R} \gtrsim 10^{10}(\beta \mathrm{GeV}) \mathrm{s}^{-1}$.

Rabi oscillations are observable if the period of the oscillations is less than the radiative life time of the excited energy level. For a hydrogen atom, the radiative lifetime of the $2 \mathrm{p}$ energy level is $1.6 \mathrm{~ns}$. For more general atoms, we still consider that the lifetime is in the ns range. Hence the Rabi oscillations are only observable if

$$
10^{-1} \lesssim \beta \mathrm{GeV},
$$

which is already excluded by particle experiments. However it might be possible to observe chameleon driven Rabi oscillations if the power of the laser were to be increased.

For currently achievable experimental setups, we conclude that the population of the excited energy level is never significant, and the system is well approximated by the weak field limit. For a GammeV-like experiment the number of photons created per unit time and per atom, $B_{\text {chameleon }}$, defined in (34), is

$$
\begin{aligned}
B_{\text {chameleon }} & \approx \frac{a^{2} m_{e}^{2} a_{0}^{2} \beta^{2} \bar{\omega}^{2} \Delta \omega}{\left(\omega_{0}-\bar{\omega}\right)^{2}+\Delta \omega^{2}} \\
& \approx \frac{10^{15} \vartheta^{2}(\beta \mathrm{GeV})^{2} s^{-1}}{\left(\frac{\omega_{0}}{\mathrm{eV}}-1\right)^{2}+10^{-14}}
\end{aligned}
$$

Where we have taken typical values for the laser spectrum with $\bar{\omega} \sim 1 \mathrm{eV}$ and a width $\Delta \omega \sim 10^{-7} \mathrm{eV}$. If the laser is not tuned to the resonance $\omega_{0} / \mathrm{eV}-1 \sim \mathcal{O}(\mathrm{eV})$. This implies

$$
B_{\text {chameleon }} \approx 10^{15} \vartheta^{2}(\beta \mathrm{GeV})^{2} s^{-1} .
$$

Recalling that the GammeV experiment is sensitive only to $\vartheta \gtrsim 10^{-9}$, we see that for a typical experiment of the GammeV type and experimentally allowed values of $\beta$ the production of photons can be significant:

$$
B_{\text {chameleon }} \gtrsim 10^{-3}(\beta \mathrm{GeV})^{2} s^{-1} .
$$

The typical lifetime of the afterglow phenomenon $1 / B_{\text {chameleon }}$ can be very large when $\beta$ is small, i.e. macroscopic and of a few seconds. In this case, the number of created photons can still be relatively large as the number of atoms in the cavity can be significant. Again considering the GammeV experiment which has a length of $6 \mathrm{~m}$ and a diameter of 3.175 $\mathrm{cm}$, and within which the pressure is $\sim 1.9 \times 10^{-3}$ torr, we find that the number of atoms within the experiment at room temperature is $\sim 2 \times 10^{18}$. The surface area of the aperture compared to the surface area of the experiment is 0.005 , so that we find that emitted photons should be detected at a rate satisfying

$$
\text { Rate of detected photons } \gtrsim 10^{13}(\beta \mathrm{GeV})^{2} s^{-1} \text {. }
$$

For sufficiently strong couplings afterglow photons are easily detectable.

Recently the GammeV collaboration reported [27] that they have seen an orange afterglow, the magnitude of which is independent of the strength of the magnetic field. 
Whilst this result is preliminary and may well be a systematic of the experiment, it is interesting to speculate, in light of the analysis of this article, that it could be the atomic emission and absorption caused by a bath of chameleons produced by the experiment.

\section{Conclusions}

The chameleon is a scalar field with environmental dependent properties. Due to a non-linear potential and a non-minimal coupling to matter the mass of the field is large in dense environments and small in diffuse ones. Such a field can have strong couplings to matter whilst still avoiding the constraints of experimental probes of gravity.

In such theories an additional coupling of the scalar field to the photon is common, which enables us to probe the chameleon model in optical experiments. The classic laboratory search for such a chameleon is an afterglow experiment where a laser is shone through a vacuum tube with a pervading magnetic field. In such an environment the photons may oscillate into chameleons, these chameleons will remain trapped in the vacuum tube. Later, after the laser has been switched off, the chameleons may oscillate back into photons. These photons are known as the afterglow.

Current afterglow experiments are able to probe the mass of the chameleon and its coupling to photons. In this article we have described how they can also be used to probe the coupling of the chameleon to matter fields. The coupling to matter means that a bath of chameleons, inside the vacuum tube of an afterglow experiment, would excite energy level transitions in atoms present in the vacuum tube. This is precisely analogous to atomic emission and absorption due to photons. If an electron is excited to a higher atomic energy level by the chameleon bath, the atom may still decay to a lower energy level by the emission of a photon. We have computed the rate at which such photons would be emitted in a typical afterglow experiment. We find that this rate can be significant, and so searches for this phenomenon can be used to constrain the chameleon to matter coupling.

Our results are particularly interesting in light of a recent detection of an orange afterglow in the GammeV experiment, which is independent of the strength of the magnetic field. However systematic explanations for this detection are yet to be excluded.

All in all, we find that the experimental detection of an afterglow phenomenon when the laser beam and the magnetic field have been turned off would lead to stringent bounds on a combination of both the coupling of chameleons to photons and matter. Depending on the values of these couplings, this afterglow phenomenon could well be a smoking gun for the possible existence of chameleons.

\section{Acknowledgments}

We would like to thank Axel Lindner, Amanda Weltman and William Wester for very helpful discussions. CB is supported by the German Science Foundation (DFG) under 
the Collaborative Research Centre (SFB) 676 and would like to thank the Institut de Physique Théorique, CEA, for their hospitality while part of this work was completed.

\section{References}

[1] C. M. Will, The confrontation between general relativity and experiment, Living Rev. Rel. 4 (2001) 4, gr-qc/0103036.

[2] J. Khoury and A. Weltman, Chameleon cosmology, Phys. Rev. D69 (2004) 044026, astro-ph/0309411.

[3] J. Khoury and A. Weltman, Chameleon fields: Awaiting surprises for tests of gravity in space, Phys. Rev. Lett. 93 (2004) 171104, astro-ph/0309300.

[4] M. A. Luty, M. Porrati, and R. Rattazzi, Strong interactions and stability in the DGP model, JHEP 09 (2003) 029, hep-th/0303116.

[5] A. Nicolis, R. Rattazzi, and E. Trincherini, The galileon as a local modification of gravity, Phys. Rev. D79 (2009) 064036, arXiv:0811.2197.

[6] C. Burrage and D. Seery, Revisiting fifth forces in the Galileon model, JCAP 1008 (2010) 011, arXiv:1005.1927.

[7] D. F. Mota and D. J. Shaw, Evading equivalence principle violations, astrophysical and cosmological constraints in scalar field theories with a strong coupling to matter, Phys. Rev. D75 (2007) 063501, hep-ph/0608078.

[8] P. Brax, C. van de Bruck, and A.-C. Davis, Compatibility of the chameleon-field model with fifthforce experiments, cosmology, and PVLAS and CAST results, Phys. Rev. Lett. 99 (2007) 121103, hep-ph/0703243.

[9] P. Brax, C. Burrage, A.-C. Davis, D. Seery, and A. Weltman, Higgs production as a probe of Chameleon Dark Energy, Phys. Rev. D81 (2010) 103524, arXiv:0911.1267.

[10] P. Brax, C. van de Bruck, A.-C. Davis, D. F. Mota, and D. J. Shaw, Testing Chameleon Theories with Light Propagating through a Magnetic Field, Phys. Rev. D76 (2007) 085010, arXiv:0707.2801.

[11] H. Gies, D. F. Mota, and D. J. Shaw, Hidden in the Light: Magnetically Induced Afterglow from Trapped Chameleon Fields, Phys. Rev. D77 (2008) 025016, arXiv:0710.1556.

[12] M. Ahlers, A. Lindner, A. Ringwald, L. Schrempp, and C. Weniger, Alpenglow - A Signature for Chameleons in Axion-Like Particle Search Experiments, Phys. Rev. D77 (2008) 015018, arXiv:0710.1555.

[13] A. S. Chou, W. Wester, A. Baumbaugh, H. R. Gustafson, Y. Irizarry-Valle, P. O. Mazur, J. H. Steffen, R. Tomlin, A. Upadhye, A. Weltman, X. Yang, and J. Yoo, Search for chameleon particles using a photon-regeneration technique, Phys. Rev. Lett. 102 (Jan, 2009) 030402.

[14] A. Upadhye, J. H. Steffen, and A. Weltman, Constraining chameleon field theories using the GammeV afterglow experiments, Phys. Rev. D81 (2010) 015013, arXiv:0911.3906.

[15] P. Brax, C. Burrage, A.-C. Davis, D. Seery, and A. Weltman, Collider constraints on interactions of dark energy with the Standard Model, JHEP 09 (2009) 128, arXiv:0904.3002.

[16] G. Rybka et. al., A Search for Scalar Chameleons with ADMX, Phys. Rev. Lett. 105 (2010) 051801, arXiv:1004.5160.

[17] C. Burrage, Supernova Brightening from Chameleon-Photon Mixing, Phys. Rev. D77 (2008) 043009, arXiv:0711.2966.

[18] C. Burrage, A.-C. Davis, and D. J. Shaw, Detecting Chameleons: The Astronomical Polarization Produced by Chameleon-like Scalar Fields, Phys. Rev. D79 (2009) 044028, arXiv:0809.1763.

[19] C. Burrage, A.-C. Davis, and D. J. Shaw, Active Galactic Nuclei Shed Light on Axion-likeParticles, Phys. Rev. Lett. 102 (2009) 201101, arXiv:0902.2320.

[20] A.-C. Davis, C. A. O. Schelpe, and D. J. Shaw, The Effect of a Chameleon Scalar Field on the Cosmic Microwave Background, Phys. Rev. D80 (2009) 064016, arXiv:0907.2672. 
[21] C. A. O. Schelpe, Chameleon-Photon Mixing in a Primordial Magnetic Field, Phys. Rev. D82 (2010) 044033, arXiv:1003.0232.

[22] A. Avgoustidis, C. Burrage, J. Redondo, L. Verde, and R. Jimenez, Constraints on cosmic opacity and beyond the standard model physics from cosmological distance measurements, arXiv: 1004.2053

[23] S. A. Levshakov et. al., Searching for chameleon-like scalar fields with the ammonia method. II. Mapping of cold molecular cores in NH3 and HC3N lines, arXiv:1008.1160

[24] G. Raffelt and L. Stodolsky, Mixing of the Photon with Low Mass Particles, Phys. Rev. D37 (1988) 1237.

[25] P. Brax, C. van de Bruck, A.-C. Davis, D. F. Mota, and D. J. Shaw, Detecting Chameleons through Casimir Force Measurements, Phys. Rev. D76 (2007) 124034, arXiv:0709.2075.

[26] M. Fox, Quantum Optics. Oxford University Press, 2006.

[27] W. Wester, "GammeV: Results and Future Plans at Fermilab." Presentation at the 6th Patras Workshop on Axions, WIMPs and WISPs, 2010. 